\title{
Psychosocial Aspects of Closed and Open Loop Insulin Delivery: \\ Closing the Loop In Adults with Type 1 Diabetes in the Home Setting
}

Running Title: Psychosocial Impact of Closed Loop at Home

Katharine D Barnard ${ }^{1}$, Tim Wysocki ${ }^{2}$, Hood Thabit ${ }^{4}$, Mark L Evans ${ }^{4}$, Stephanie Amiel, ${ }^{5}$ Simon Heller 6 , Amanda Young ${ }^{3}$, Roman Hovorka ${ }^{4}$ on behalf of Angela Consortium

1. $\mathrm{HDH}$, Faculty of Medicine, University of Southampton

2. Center for Health Care Delivery Science, Nemours Children's health System, Jacksonville, Florida, USA

3. National Institute for Health Research, Evaluation, Trials and Studies Coordinating Centre (NETSCC), University of Southampton, Southampton, UK

4. Wellcome Trust/ MRC Institute of Metabolic Science, University of Cambridge and NIHR Cambridge Biomedical Research Centre, Cambridge, UK

5 Division of Diabetes and Nutritional Sciences, King's College London and King's College Hospital NHS Foundation Trust

6. Department of Human Metabolism, School of Medicine and Biosciences, University of Sheffield.

Corresponding author:

Katharine Barnard CPsychol AFBPsS

E: k.barnard@soton.ac.uk

Word count: 3212

Funding: Diabetes UK

Conflict of interest: None

Novelty Statement:

- Closed loop technology has specific psychosocial benefits supporting optimal self-management in adults with type 1 diabetes

- Advances in technology are associated with greater usability with further development work necessary to improve connectivity

- Future research is required to determine the holistic impact of closed loop as a realistic therapy choice for people with type 1 diabetes 


\section{Abstract:}

Aim: to explore 1) psychosocial experiences of closed loop technology and 2) compare ratings of closed and open loop technology for adults with type 1 diabetes taking part in a randomized crossover study.

Methods: Adults age $>18$ years on insulin pump therapy were recruited to received real-time continuous glucose monitoring (CGM) with overnight closed-loop or real-time CGM alone (open loop) followed by the alternative treatment randomly, at home for 4-weeks unsupervised. Participants were invited to share their views in semi-structured interviews. Impact of the closed loop technology, positive and negative aspects of living with the device overnight, alongside hopes and anxieties were explored.

Results: Participants: 24 adults, mean age 43(SD 12), 54\% male. Interview duration 12-46 minutes (mean 26 minutes). Content and thematic analysis revealed key positive themes: Improved blood glucose control $(n=16)$; Reassurance/reduced worry $(n=16)$; Improved overnight control leading to improved daily functioning and diabetes control $(n=16)$; Improved sleep $(n=8)$. Key negative themes: Technical difficulties $(n=24)$; Intrusiveness of alarms $(n=13)$; Size of equipment $(n=7)$. Twenty participants would recommend the closed loop technology.

Conclusions: Closed loop has positive effects when it works in freeing participants from the demands of self-management. The downside for those who had more negative views was generally down to technical difficulties particularly around the pump and 'connectivity' which should hopefully improve. Future research should continue to explore acceptability as a realistic therapy option, taking account of user concerns as new systems are designed. Failure to do this may reduce the eventual utility of new systems. 


\section{Background}

Closed loop technologies, sometimes referred to as an artificial pancreas (AP), are developing rapidly with successful diabetes camp [1] and at home/outpatient trials [2-6]. Closed loop systems have demonstrated clinical efficacy resulting in tighter overnight glycaemic control without increased frequency or severity of hypoglycemia $[3,4]$. With the potential to provide a realistic treatment option for people with type 1 diabetes (T1DM), the usability and impact on psychosocial outcomes will be crucial factors in determining the realization of benefit from use of this technology [7].

Maintenance of optimal glycaemia overnight is particularly challenging, with nocturnal hypoglycaemia common, representing a critical problem in the management of type 1 diabetes (T1DM) and causing heightened anxiety affecting psychosocial functioning [8]. The risk of hypoglycaemia represents a major obstacle to the achievement of optimal blood glucose levels [9]. Early overnight closed loop research has show positive results in achieving optimal glycaemia without increased risk or frequency of hypoglycaemia for adolescent participants [3,4].

The current open-label, multicentre, randomized crossover study recruited participants from three centres in the UK. Participants aged 18 years or older with type 1 diabetes were randomly assigned to receive four weeks of overnight closed-loop insulin delivery (using a model-predictive control algorithm to direct insulin delivery), then four weeks of insulin pump therapy with real-time continuous glucose monitoring (open loop phase), or vice versa. The primary outcome was time spent in the target glucose range of 3.9-8.0 $\mathrm{mmol} / \mathrm{L}$ between midnight and 0700 hours. Analyses were by intention to treat [4].

The purpose of this sub-study, alongside the main trial, was to explore 1) psychosocial experiences of closed loop technology and 2) compare ratings of closed and open loop technology for adults with type 1 diabetes taking part in a randomized crossover study. 


\section{Methods and Participants}

The main aims and methodology of the trial have been reported in detail elsewhere [4]. In brief, alongside efficacy and safety outcomes of closed loop technology in this open-label, single-centre, randomised two period cross-over study (12 completing closed loop phase first), the utility of the technology was evaluated. Inclusion criteria were type 1 diabetes (WHO criteria), C-peptide negative, aged 18 years or older, insulin-pump therapy for at least 3 months, knowledge of insulin self-adjustment, undertaking of glucose self-monitoring at least four times daily, and $\mathrm{HbA} 1 \mathrm{c}$ of $10 \%(86 \mathrm{mmol} / \mathrm{mol})$ or lower. Exclusion criteria were established nephropathy, neuropathy or proliferative retinopathy, total daily insulin dose of $2.0 \mathrm{U} / \mathrm{kg}$ or greater, regular use of continuous glucose monitoring within 1 month before enrolment, severe visual or hearing impairment, pregnancy, or breastfeeding. The study protocol was approved by the East of England Central Cambridge Ethics Committee. The study included a mixed methods approach, using psychosocial questionnaires and semi-structured interviews to evaluate participants' perceptions of lifestyle change, diabetes management and fear of hypoglycaemia.

\section{Questionnaire Data}

At study entry and again at the end of two interventions, each participant completed the Diabetes Technology Questionnaire (DTQ). This is a 30-item measure of the impact of, and satisfaction with, technological tools that may be used in the management of T1DM [8]. Participants were asked to rate their agreement or disagreement with statements regarding the specific complement of diabetes technologygies in use at that measurement point (ie, real time continuous glucose monitoring alone or meter, pump, continuous glucose monitor, closed loop with real time continuous glucose monitoring-AP). Individual items are scored separately for each of the two columns of response options, yielding separate total scores for "Is this a problem now?" (Current) and "How has it changed compared to your treatment before the study?" (Change). The DTQ was administered at baseline and at the end of the two 28-day crossover periods. Statistical analysis was conducted using SPSS (version 21). Analysis was conducted using descriptive statistics and 
repeated measures including t-test and ANOVA with a statistical significance threshold of $0.05 \%$. Statistical analysis was conducted using SPSS (version 21).

The DTQ yields separate scores for 'Current' (How much is this a problem now?) and 'Change' (How has it changed compared to before the study?) subscales. At Baseline, only the "Current" items were administered, while at the conclusion of each crossover period, both the "Current" and "Change" items were administered. After reverse scoring of some items, higher scores are indicative of more favourable satisfaction and impact ratings of the technology. Based on participant responses in the present study, internal reliability (Cronbach's Alpha Coefficient) remained above 0.91 for the DTQ "Current" items and above 0.94 for the DTQ "Change" items throughout the study. Previous unpublished data from a sample of 115 adolescents and parents yielded similar psychometric data.

For the present study, the DTQ was modified to include a 9-item "User Friendliness" section for each of four possible technological components of the participants' diabetes regimens (glucose meter, insulin pump, continuous glucose monitor, closed loop insulin delivery system). Participants rated the complete package of technology they had been using as well as each component that was part of their current regimen along each dimension of user friendliness on a 5 point scale with 1 = "Terrible" and $5=$ "Excellent".

\section{Semi-Structured Interviews}

Semi-structured interviews to explore participants' perceptions of the impact of the technologies on their experiences were designed in collaboration with the clinical research team. Factors including lifestyle change, diabetes management, safety and impact on their ability to carry out their usual daily routines were discussed. The interview schedule was then piloted on four potential participants for usability, relevance, and acceptability. These participants were not included in the study. The feedback was positive with minor revisions suggested, and the interview schedule was revised in line with this. All participants taking part in the study were invited to participate in the interview study and all elected to do so. 
On completion of each intervention, participants were invited to partake in an audio-recorded telephone interview conducted by KDB. All interviews were conducted within 2 weeks of the end of the trial. Audiotapes were transcribed with all identifying details removed. Following transcription, KDB and AJY performed independent thematic analyses, reading each participant's interview in full before performing cross-comparisons to identify continuities and differences between accounts. This analysis was used to develop a coding framework which captured original research questions and emerging findings [11].

Content analysis focused on the number/frequency of 'instances', their context, meaning and whether they were common across participants. Free text analysis concentrated on identifying key themes arising with a view to understanding the experiences of participants, exploring connections between themes and identifying how the technology affected everyday living and factors important to quality of life in ways that are important to participants.

\section{Results}

Twenty-four adults, mean age 43 (SD 12) years, 54\% male, HbA1c 65 (9) $\mathrm{mmol} / \mathrm{mol}\left[8.1 \%(0.8), \mathrm{BMl} 26.0(3.5) \mathrm{kg} / \mathrm{m}^{2}\right.$, duration of diabetes 29 (11) years, participated in the interviews. Interview duration ranged from 12 to 46 minutes (mean 26 minutes). Twenty-two participants completed the trial, two participants were classified as having a shortened study period (where the closed loop technology safety mechanism instigated reversion to routine insulin pump therapy), one of whom would have preferred to continue. All data are reported collectively.

Questionnaire Data: DTQ data at one or more measurement points were missing for three participants. Mean DTQ item scores during each phase of the study are shown in Table 1 at Baseline (DTQ "Current" items only) and after the Closed Loop and Open Loop phases (DTQ "Current" and "Change" items). The mean item scores were all above 3.0, indicating overall favourable 
ratings of impact of and satisfaction with the diabetes technology just recently used by the participants. Although the DTQ Current item scores appeared to be higher (more favorable) after the Open Loop phase, these scores did not differ significantly between any of the three conditions. The same was true of the comparison DTQ "Change" scores between the Closed Loop and Open Loop conditions. Two-tailed t-tests for related means comparing DTQ "Current" total scores at Baseline vs Open Loop ( $t=-1.48$; $p<0.16$ ), Baseline vs Closed Loop ( $t=0.47 ; \mathrm{p}<0.65$ ), and Closed Loop vs Open Loop ( $t=-1.24 ; p<0.23$ ) all failed to reach statistical significance. The same was true of the comparison of DTQ "Change" total scores following the Closed Loop and Open Loop phases $(t=-1.18 ; p<0.26)$.

\section{(Insert Table 1 about here)}

User Friendliness ratings of each technological component of participants' current diabetes regimens were evaluated by examining the percentages of responses in the "Good" or "Excellent" categories. The most important of these results, presented in Table $3-\underline{2}$ below, concern participants' ratings of the closed loop insulin delivery system after the Closed Loop phase of the study. The results show that participants were least satisfied with the use of the closed loop system during physical activity and bathing and regarding appearance issues created by use of the system (mean ratings in the "Poor" range). The participants were most satisfied with the closed loop system regarding ease of start-up and calibration, the instructions, manual and technical support that were provided, and performance accuracy/reliability (mean ratings in the "Good" range). None of the items yielded a mean rating above 4 (Good) on the 5-point response scale.

\section{(Insert Table 2 about here)}

These results also indicated relatively fewer "Good" or "Excellent" ratings of the insulin pumps used during the Open (27.8\%) and Closed (29.4\%) Loop phases compared to baseline (77.2\%). 
Eight participants directly spoke about their improved sleep as a consequence of using the closed loop system. Reassurance and peace of mind associated with the closed loop technology were frequently cited as benefits $(n=16)$. Being able to worry less about diabetes and blood glucose levels, as well as the security of knowing that the technology was 'taking over for a while' contributed to this reassurance.

Sixteen participants directly commented on having improved blood glucose levels as a result of using the closed loop system, reporting that 'waking up on a good number' contributed to improved blood glucose control into the following day, providing a 'better start' to the day rather than waking up and immediately having to deal with a hypo or hyperglycaemia.

\section{(Insert Table 3 about here)}

In response to the question 'what would you change about the closed loop system?' thirteen participants said 'the equipment', specifically, six would make it smaller and seven would make it more portable, including increasing the Bluetooth range, i.e. improving connectivity.

In response to 'What was the impact on your daily life?', eight participants reported the closed loop could 'do a better job than I could' reporting that having improved nocturnal glycaemic level meant they had a better day. Five reported the positive impact of improved blood glucose control, saying they 'felt better', 'worried less' and 'had increased confidence'. Negative impacts reported were the system being 'inconvenient' $(n=4)$ and it 'restricting movement' $(n=1)$, with another participant saying the whole experience was 'really hard'.

Twenty participants would recommend the closed loop technology to a friend with type 1 diabetes, one participant couldn't answer this question and three would not recommend it. 
When asked for their preference between closed loop and CGM, twelve participants chose the closed loop system, four the CGM, two did not answer and six participants were unable to provide a preference for a number of reasons:

- 'no preference - Closed Loop took a little more effort'

- 'Closed Loop isn't quite there yet...it required more time'

- 'Open Loop [CGM] you are still controlling yourself...Closed Loop took more effort'

- 'Open Loop [CGM] is a useful system...takes more time. Closed Loop takes control away...it was quite liberating'

These responses are diametrically opposite in the sense that both quotes refer to the autonomy of the Closed Loop, with one person finding this problematic and the other really liking it.

\section{Key Positive Experiences}

Table 3 illustrates the positive comments received. The enthusiasm and strength of feeling expressed by some participants about their experience of the Closed Loop were significant. 'Brilliant', 'sad to finish', 'felt half way human', 'I was a nicer person to be around', 'I was more effective, more productive' and 'I felt normal' were all comments made by participants. None of the participants regretted taking part in the trial and all had something positive to say about their experience of being involved. However, three participants who completed the trial felt that participation had been challenging at times, saying 'it took over my life' (005), "I was sick to death of it' (018) and "it was fragile, laborious and had a major impact on my routine." (023).

Potential reduction of long-term complications (via improved blood glucose control overnight) were reported by three participants. 'Improved health', 'more energy', feeling 'liberated' and 'generally feeling better' were cited as benefits by participants. Similarly, 'improved sleep' was reported by six participants contributing to general feelings of well-being. One participant 
commented on the change in their partner's attitude towards their diabetes saying 'it made him [partner] much more aware of how difficult it is controlling diabetes .... made him much more caring' (018)

Furthermore, reassurance, reduced worry and peace of mind were associated with using the closed loop $(n=16)$, with a better understanding of diabetes $(n=3)$ and seeing how 'it' responded to different situations was helpful $(n=1)$. Nineteen participants felt safe with the device, particularly once their initial anxiety about whether it would perform on the first day or two, and were confident that it "was OK". See table 3 for detail.

The concept of the technology and the prospect of 'how good it could be' in the not too distant future, moving towards a fully automated system and associated possibilities was reported as a benefit by nine participants $(n=9)$, something that was expressed by parents of adolescents taking part in the previous study. Several participants said that they had taken part to help 'further the cause' of diabetes research and hoping to benefit other people as well as themselves in the future. There was also widespread acknowledgement that this is cutting edge research and so some teething difficulties were inevitable.

\section{(Insert table 4 about here)}

Key Negative Experiences

Table 4 illustrates the key negative experiences reported.

\section{(Insert Table $\underline{4}$ about here)}

All participants reported having experienced some technical or usability difficulties with the equipment, however these were most often associated with the insulin pump and continuous glucose monitor than with the Closed Loop technology itself. Common problems included connection difficulties, poor battery life, sensors not sticking very well or getting in the way /being too large. See table 4 for detail. 
The alarms were also a problem for several participants $(n=13)$, particularly when they became intrusive by waking up other family members or caused repeated sleepless nights. Feelings of powerlessness were reported in terms of the alarm sounding, corrective action being taken promptly but then the alarms continuing to sound every 20 minutes (or so) as blood glucose levels were returning to a safe range. Interestingly, participants did not distinguish between 'real' alarms or 'false' alarms, so it is not possible to know whether the alarms were always appropriate. Two participants did however report that the alarms were a positive aspect of the technology because they gave them a greater awareness of hypo- and hyper- glycaemia and were able to act quickly to treat them.

Seven participants commented on the size of the equipment, specifically the lack of portability of the laptop, the size of the sensors, having to carry so many things but lacking somewhere to put them. For those participants who used both first generation and second generation sensors, the reduced size of the second generation sensors was reported favourably.

Three participants reported the inconvenience of using the equipment, finding it hard to fit it into their lifestyles. Another participant reported increased anxiety because of the technology, with a further three participants reporting more episodes of hypoglycaemia than usual whilst on the closed loop. For one of these, it 'knocked my confidence'.

\section{Discussion}

The impact of the tested Closed Loop technology was positive for most participants' experience $(n=21)$, outweighing reported downsides which were often described as 'teething' problems with the technology.

Closed Loop technology represents cutting edge technological research in the treatment of type 1 diabetes, with technological advancements facilitating a progression to home-based trials. There is scant evidence however on whether it will meet people's needs in the context of usability and psychosocial functioning. Alongside biomedical and cost-effectiveness 
benefits, the technology must be usable, safe and beneficial from a psychosocial perspective if people are going to be able to embrace it as a realistic therapy option.

Participants' impact and satisfaction ratings after use of Closed Loop or Open Loop technology, as measured by the DTQ, revealed that, overall, participants were moderately favourably disposed toward both insulin delivery systems. Of the 30 DTQ items, 20 differences scores favored Closed Loop, 7 difference scores favored Open Loop and 3 difference scores were 0 , favoring neither alternative.

Analysis of User Friendliness of the Closed Loop system revealed moderately positive opinions of the technology except for items measuring appearance concerns and difficulties using the system during physical activity and bathing. Otherwise, the usability ratings were moderately positive (in the "Good" range), revealing aspects of the Closed Loop system needing further improvement. This was also reflected in the interviews, where participants reported challenges transferring to the study insulin pump which was different to the one they usually wore.

Key themes, both positive and negative, were similar to that reported for adolescents with type 1 diabetes [11], with widespread optimism for future technological developments and potential availability commercially in the near future. Usability challenges perhaps had a greater impact on daily living than those reported by adolescents, contributing to less enthusiasm for the technology than was reported by parents in previous research. Participants in the current study reported hoping the closed loop would be available for younger generations with type 1 diabetes and were thinking about potential availability as their own children grew up.

Technological advances in diabetes management have been rapid over recent years and have been met with mixed reviews. Insulin pump therapy and continuous glucose monitoring devices have been both positively and 
negatively associated with quality of life [12,13]. Whilst benefits of these technologies are widely reported, the reality of having to live with a device constantly over a period of time can be challenging. It could be argued that participants in closed loop research are 'highly motivated' [2] and perhaps not representative of the wider T1DM population. Thus it is important to consider that interruptions to daily living and the requirement to dedicate more time than desired on diabetes self-management take their toll on the lived experience and quality of life. Technological development should ensure the psychosocial impact of devices is fully considered.

Limitations of the current study include the low participant numbers and the fact that the trial was powered on time spent in target glucose range, so lacked statistical power to detect significant differences in psychosocial functioning between the two arms. Similar challenges have been found in previous research [5] where anxiety and quality of life was not assessed due to the short duration of the intervention. The interviews were designed to add depth and psychosocial context to the biomedical results of the main trial and as such were not designed as a detailed qualitative evaluation that would have required considerably longer interviews and phenomenological analysis.

\section{Conclusions}

The development and refinement of closed loop technology is moving apace. Representing cutting edge therapy for the treatment of type 1 diabetes, the biomedical benefits are matched by psychosocial outcomes. The additional information provided from the open loop technology was liked by participants however technical glitches were reported drawbacks. The closed loop device has major positive effects when it works in freeing patients from some of the demands and challenges of self-management. The downside for those who had more negative views was generally down to technical difficulties particularly around the pump and the 'connectivity' that should hopefully improve. Future research should continue to explore the holistic acceptability of closed loop as a realistic therapy option and take account of user concerns as new systems are designed. Failure to do this may reduce the eventual utility of new systems. 
Online appendix:

Angela Consortium comprises additionally to article co-authors investigators from King's College London: Marietta Stadler, Andrew Pernet, Pratik Choudhary; University of Cambridge: Lalantha Leelarathna, Janet M. Allen, Kavita Kumareswaran, Malgorzata E. Wilinska, Marianna Nodale, David B. Dunger, Josephine Hayes, Arti Gulati, Gopal Kotecha; and University of Sheffield: Alexandra Lubina-Solomon, Emma Walkinshaw, Ahmed lqbal, Chloe Nisbet

\section{$\underline{\text { References }}$}

1. Ly TT, Breton MD, Keith-Hynes P, De Salvo D, Clinton P, Benassi K, Mize B, Chernavvsky D, Place J, Wilson DM, Kovatchev BP, Buckingham BA. Overnight Glucose Control with an Automated, Unified Safety System in Children and Adolescents with Type 1 Diabetes at Diabetes Camp. Diabetes Care May 30, 2014 epub ahead of print

2. Nimri R, Muller I, Atlas E, et al. Night glucose control with MD-Logic artificial pancreas in home setting: a single blind, randomized crossover trial-interim analysis. Pediatr Diabetes 2014; 15: 91-99.

3. Hovorka R, Elleri D, Thabit $\mathrm{H}$, et al. Overnight closed-loop insulin delivery in young people with type 1 diabetes: a free-living, randomized clinical trial. Diabetes Care 2014; 37: 1204-11.

4. Thabit $H$, Lubina-Solomon A, Stadler M, Leelarathna L, Walkinshaw E, Pernet A, Allen J, Iqbal A, Choudhary P, Kumareswaran K, Nodale M, Nisbet C, Wilinska M, Barnard KD, Dunger DB, Heller SR, Amiel SA, Evans ML and Hovorka R. Home use of closed-loop insulin delivery for overnight glucose control in adults with type 1 diabetes: a 4-week, multicenter, randomized crossover study. The Lancet S22138587(14)70114-7 16 June 2014

5. Phillip M, Battelino T, Atlas E, Kordonouri O, Bratina N, Miller S, Biester T, Stefanija MA, Muller I, Nimri R, Danne T. Nocturnal glucose control with an artificial pancreas at a diabetes camp. New England Journal of Medicine 2013 Feb 28;368(9):824-33

6. Russell SJ, El-Khatib FH, Sinha M, Magyar KL, McKeon K, Goergen LG, Balliro C, Hillard MA, Nathan DM, Damiano ER. Outpatient glycemic controil with a bionic pancreas in Type 1 diabetes. New England Journal of Medicine 2014 Jun 15 epub ahead of print

7. Oliver N, Heller S, Amiel S, Evans MA, Hovorka R, Barnard KD. The importance of patient-centred outcomes in artificial pancrease research. Comment on Doyle FJ et al Closed-Loop Artificial Pancreas Systems: Engineering the Algoriths. Diabetes Care 2014 Apr 37(5):11917. Accepted Diabetes Care June 2014 in press

8. Barnard, K.D., Royle, P., Thomas, S., and Waugh, N.R. Systematic Literature Review into Parental Fear of Acute Hypoglycaemia. BMC Paediatrics - July 2010; 10:50 Abstracted in DARE www.crd.york.ac.uk/crdweb/ 
9. Cryer, P.E. The barrier of hypoglycaemia in diabetes. Diabetes 57:3169-3176, 2008

10. Neuendorf, K.A (2002) The content analysis handbook. SAGE publications, USA

11. Barnard KD, Wysocki T, Allen JM, Elleri D, Thabit $H$, Leelarathna L, Gulati A, Nodale M, Dunger DB, Tinati T and Hovorka R. Closing the loop overnight at home setting: psychosocial impact for adolecents with type 1 diabetes and their parents. BMJ Open Diab Res Care 2014;2:e000025 doi:10.1136/bmjdrc-2014-000025

12. Barnard, K.D., Lloyd, C.E. and Skinner, T.C. "Systematic Literature Review into Quality of Life Issues Surrounding Insulin Pump Use in Type 1 Diabetes". Diabetic Medicine, 2007

13. Juvenile Diabetes Research Foundation Continuous Glucose Monitoring Study Group. Factors Predictive of Use and of Benefit from Continuous Glucose Monitoring in Type 1 Diabetes. Diabetes Care, 2009 DOI: $10.2337 / \mathrm{dc} 09-088$ 
Table 1. Mean (SD) DTQ scores for the Current and Change items at Baseline and following the Closed Loop and Open Loop phases of the study.

\begin{tabular}{|l|c|c|c|}
\hline DTQ Scores & $\begin{array}{c}\text { Baseline } \\
(n=22)\end{array}$ & $\begin{array}{c}\text { Open Loop } \\
(n=19)\end{array}$ & $\begin{array}{c}\text { Closed Loop } \\
(n=20)\end{array}$ \\
\hline Current & $3.66(0.53)$ & $3.89(0.52)$ & $3.63(0.64)$ \\
\hline Change & & $3.22(0.44)$ & $3.24(0.63)$ \\
\hline
\end{tabular}

Table 2. Participants' ratings of the closed loop insulin delivery system

\begin{tabular}{|l|l|l|}
\hline User Friendliness Item & Mean & SD \\
\hline System Size, Weight, Appearance, Fashion Issues & 2.29 & 0.72 \\
\hline Ease of Start-Up, Calibration, etc. & 3.95 & 1.02 \\
\hline Battery Life and Ease of Replacement & 3.05 & 1.39 \\
\hline Variety and Flexibility of Functions & 3.44 & 1.29 \\
\hline Instructions, Manual and Technical Support & 3.95 & 0.81 \\
\hline Screen Information and Reports & 3.35 & 1.14 \\
\hline Alarm Functions & 3.38 & 1.20 \\
\hline Use During Sports, Exercise, Bathing & 2.00 & 1.28 \\
\hline Accuracy and Reliability of Performance & 3.79 & 1.03 \\
\hline
\end{tabular}


Table 3: Key Positive Themes

\begin{tabular}{|c|c|}
\hline Theme and participant examples & ID \\
\hline \multicolumn{2}{|l|}{ Reassurance/peace of mind } \\
\hline $\begin{array}{l}\text { "The reassurance, it was comforting knowing it was there" ... "My } \\
\text { blood sugars were more stable than they normally are". } \\
\text { "I went to bed confident that they were better than they normally } \\
\text { would be". } \\
\text { "Not worrying about my blood sugars and feeling it was its } \\
\text { responsibility, not mine". }\end{array}$ & 022 \\
\hline "I didn't have to worry" & 010 \\
\hline "I didn't go to sleep worrying about a hypo" & 014 \\
\hline "I didn't need to worry as it looked after you" & 016 \\
\hline $\begin{array}{l}\text { "You could wake up, look at this diagram of the profile of your blood } \\
\text { sugar and it would be dead level, erm absolutely constant } \\
\text { throughout the night and that makes you feel better". }\end{array}$ & 006 \\
\hline $\begin{array}{l}\text { "It was pretty good .... It was the best I have had for several years". } \\
\text { "I didn't go to sleep worrying about having a hypo or not waking up". } \\
\text { "I knew that it would correct this, it was like a break". }\end{array}$ & 014 \\
\hline $\begin{array}{l}\text { "It was brilliant ... it allowed me to stop worrying about a lot of things } \\
\ldots \text {... }\end{array}$ & 013 \\
\hline \multicolumn{2}{|l|}{ General Positive Comments } \\
\hline $\begin{array}{l}\text { "In the } 2 \text { weeks I was using it, it was brilliant". } \\
\text { "The principle of it is wonderful [if it lived up to expectations]". }\end{array}$ & 017 \\
\hline $\begin{array}{l}\text { "I didn't realize how much better I could feel". } \\
\text { "I've never felt better in my adult life .... I felt normal, more fund to } \\
\text { be around". } \\
\text { "It made me much more effective, more productive and much nicer } \\
\text { to be around". }\end{array}$ & 003 \\
\hline $\begin{array}{l}\text { "I felt really emotional ... like I was on the brink of something". } \\
\text { "The whole thing was brilliant". }\end{array}$ & 012 \\
\hline $\begin{array}{l}\text { "The whole thing was brilliant". } \\
\text { "When it finished I was really sad". }\end{array}$ & 012 \\
\hline $\begin{array}{l}\text { "Very impressed, I enjoyed it very much and wish it hadn't stopped". } \\
\text { "If I could get over my dislike of the pump, that would be the worse } \\
\text { thing about it, it would be coming off it and giving the system back". }\end{array}$ & 004 \\
\hline "Once you got used to it, it was really good". & 007 \\
\hline \multicolumn{2}{|l|}{ Better sleep } \\
\hline $\begin{array}{l}\text { "It did away with the intrusiveness and not having to wake up and } \\
\text { check blood glucose levels" }\end{array}$ & 017 \\
\hline $\begin{array}{l}\text { "Better sleep pattern ...When it worked it was fantastic ... I had } 8 \\
\text { hours uninterrupted which is fantastic". }\end{array}$ & 009 \\
\hline
\end{tabular}




\begin{tabular}{|c|c|}
\hline "Better sleep" & 011 \\
\hline $\begin{array}{l}\text { "You were in the morning at a good level to start with". } \\
\text { "You didn't worry when you went to sleep because you knew it was } \\
\text { going to look after you". }\end{array}$ & 016 \\
\hline Wake up in the morning feeling normal & 020 \\
\hline \multicolumn{2}{|l|}{ Improved blood glucose control } \\
\hline "Better insight into controlled whole diabetes" & 018 \\
\hline "It managed to maintain blood glucose levels" & 004 \\
\hline "I only had one overnight hypo" & 010 \\
\hline $\begin{array}{l}\text { "It was brilliant! All conversations are 'we don't know what's } \\
\text { happening at night .... Now it's good overnight and we don't have to } \\
\text { think about it. I slept so much better." } \\
\text { "Knowing that for } 8 \text { hours a day, you have good control." } \\
\text { "There was nothing bad about the algorithm [but the equipment was } \\
\text { still quite raw]." }\end{array}$ & 001 \\
\hline "My blood glucose was perhaps better controlled". & 005 \\
\hline $\begin{array}{l}\text { "I was really impressed with how it stabilized my blood sugar, it was } \\
\text { pretty much spot on". }\end{array}$ & 011 \\
\hline $\begin{array}{l}\text { "Waking up in the morning and seeing your blood sugars in a } \\
\text { straight line for about } 12 \text { hours which was fantastic". } \\
\text { "I can't describe that feeling but it was brilliant. It really is amazing". }\end{array}$ & 019 \\
\hline $\begin{array}{l}\text { "My sugars were a lot better in the morning ... it does what it says } \\
\text { on the tin". } \\
\text { "The technology is coming forward and research is coming forward } \\
\ldots . \text { There will be solutions to the issues". }\end{array}$ & 008 \\
\hline $\begin{array}{l}\text { "The concept .. moving towards an artificial pancreas optimizing } \\
\text { people's sugar controls to improve the levels to avoid high } \\
\text { readings". } \\
\text { "It was generally doing at least as good if not a better job than I } \\
\text { would be doing myself". }\end{array}$ & 002 \\
\hline "Wake up with perfect blood glucose levels, good start to the day" & 021 \\
\hline \multicolumn{2}{|l|}{$\begin{array}{l}\text { Improved blood glucose control overnight led to improved } \\
\text { daytime control }\end{array}$} \\
\hline "I was impressed how it controlled things during the day" & 013 \\
\hline "Better during the day as it's being controlled better" & 015 \\
\hline "Feeling better in the morning" & 011 \\
\hline $\begin{array}{l}\text { "My control overnight almost all of the time was excellent." } \\
\text { "I would pretty much always wake up with a perfect blood sugar } \\
\text { which was then a really good way to start your day". }\end{array}$ & 021 \\
\hline
\end{tabular}


Table 4: Key Negative Themes

\begin{tabular}{|c|c|}
\hline Theme and participant examples & ID \\
\hline \multicolumn{2}{|l|}{ Alarms beeping frequently } \\
\hline 'absolutely dreadful ... sick to death of it' & 018 \\
\hline 'It bleeped a lot at night, the alarms' & 015 \\
\hline 'Alarming all the time' & 021 \\
\hline 'Problems with the companion waking me up' & 005 \\
\hline "The alarms .... Everybody knows". & 001 \\
\hline $\begin{array}{l}\text { "There were so many times when for a variety of reasons it kept } \\
\text { me awake all night because it was alerting or it didn't seem to } \\
\text { work". } \\
\text { "The worst thing for me was not knowing whether what was } \\
\text { happening was down to operator incompetence or the technology". }\end{array}$ & 009 \\
\hline $\begin{array}{l}\text { "There were a couple of stressful moments ... it was not as robust } \\
\text { or resilient as I thought it would be". } \\
\text { "The alarms were very intrusive". }\end{array}$ & 003 \\
\hline "The alarms would go off [when setting up the system]". & 008 \\
\hline $\begin{array}{l}\text { "The alarms". } \\
\text { "The whole system is too big ... you couldn't walk around with it". }\end{array}$ & 007 \\
\hline $\begin{array}{l}\text { "The alarms ... connecting them altogether was a problem really" } \\
\text { "I found it difficult with my shift patterns" } \\
\text { "The equipment really ..." }\end{array}$ & 015 \\
\hline $\begin{array}{l}\text { "It was awful ... it wasn't for me at all". } \\
\text { "The inconvenience in the evening, the bleeping just going off . } \\
\text { its sleep deprivation for me" }\end{array}$ & 020 \\
\hline $\begin{array}{l}\text { "It got a bit confused .. it bleeped at night a couple of times and } \\
\text { kept losing contact". }\end{array}$ & 012 \\
\hline \multicolumn{2}{|l|}{ Technical/Usability difficulties } \\
\hline Problems with the hardware & 018 \\
\hline Connecting the devices together was a problem & 015 \\
\hline It kept losing contact & 012 \\
\hline "The calibration at night". & 007 \\
\hline It's noisy, technical setting it up and the range is too short & 016 \\
\hline "It's too easy to turn off by mistake". & 004 \\
\hline $\begin{array}{l}\text { "I had a lot of trouble with the transmitter". } \\
\text { "The terrible row ..." } \\
\text { "The first week I felt sleep deprived". }\end{array}$ & 005 \\
\hline $\begin{array}{l}\text { "The equipment wasn't very easy to use" [talking mostly about } \\
\text { CGM and pump] }\end{array}$ & 006 \\
\hline $\begin{array}{l}\text { "The noise from the hardware ... the fan .. next to the bed". } \\
\text { "I was wary of it losing its connection". }\end{array}$ & 010 \\
\hline $\begin{array}{l}\text { "I had to sleep very close to the thing which meant I didn't have } \\
\text { much room to move" }\end{array}$ & 016 \\
\hline
\end{tabular}




\begin{tabular}{|c|c|}
\hline $\begin{array}{l}\text { "If I turned over the sensor was too far away from the CAD and it } \\
\text { got detached which was the worse thing" } \\
\text { "It's quite restrictive" }\end{array}$ & \\
\hline "The connectivity .. the blue tooth" & 013 \\
\hline $\begin{array}{l}\text { "You feel a bit battered by the end of it ... fit your whole life around } \\
\text { a machine that isn't working that well". }\end{array}$ & 019 \\
\hline \multicolumn{2}{|l|}{ Night-time hypos } \\
\hline $\begin{array}{l}\text { "Absolutely dreadful". } \\
\text { "I was really angry by the end of it ... I had a lot of problems with } \\
\text { the hardware". } \\
\text { "The whole thing overwhelmed me I have to say". } \\
\text { "You couldn't image what was going to go wrong next" } \\
\text { "Whilst I was on the Closed Loop ... I was really worried because I } \\
\text { kept going hypo" } \\
\text { "I found it made me really anxious" }\end{array}$ & 018 \\
\hline More night hypos on the Closed Loop & 005 \\
\hline \multicolumn{2}{|l|}{ Too big } \\
\hline $\begin{array}{l}\text { "Not very portable .... So much equipment you need to be using } \\
\text { for the Closed Loop" }\end{array}$ & 011 \\
\hline Needs to be smaller & 014 \\
\hline It's bulky & 016 \\
\hline $\begin{array}{l}\text { ".. limited by the actual technologies available ..." } \\
\text { "You have to be within reasonable range for the transmitter to work } \\
\text { ".." } \\
\text { "All the communication between the algorithm and equipment ..." }\end{array}$ & 002 \\
\hline "Making it smaller would be the biggest thing" & 014 \\
\hline
\end{tabular}

